\title{
Altered resting state EEG in chronic pancreatitis patients: toward a marker for chronic pain
}

This article was published in the following Dove Press journal: Journal of Pain Research

22 November 2013

Number of times this article has been viewed

\author{
Marjan de Vries' \\ Oliver HG Wilder-Smith ${ }^{2}$ \\ Marijtje LA Jongsma ${ }^{3}$ \\ Emanuel $\mathrm{N}$ van den Broeke' \\ Martijn Arns ${ }^{5,6}$ \\ Harry van Goor' \\ Clementina M van Rijn ${ }^{4}$ \\ 'Department of Surgery, Radboud \\ University Nijmegen Medical \\ Centre, Nijmegen, The Netherlands; \\ ${ }^{2}$ Department of Anesthesiology, \\ Pain and Palliative Care, Radboud \\ University Nijmegen Medical \\ Centre, Nijmegen, The Netherlands; \\ ${ }^{3}$ Behavioral Science Institute, \\ Radboud University Nijmegen, \\ Nijmegen, The Netherlands; \\ ${ }^{4}$ Donders Institute for Brain, \\ Cognition and Behavior, Radboud \\ University Nijmegen, Nijmegen, \\ The Netherlands; ${ }^{5}$ Research Institute \\ Brainclinics Nijmegen, Nijmegen, \\ The Netherlands; ${ }^{6}$ Department of \\ Experimental Psychology, Utrecht \\ University, Utrecht, The Netherlands
}

Correspondence: Marjan de Vries

Department of Surgery, Radboud

University Nijmegen Medical Centre,

PO Box 910I, 6500 HB Nijmegen,

The Netherlands

$\mathrm{Tel}+31243610903$

Fax +3। 24354050 I

Email m.devries@chir.umcn.nl
Objectives: Electroencephalography (EEG) may be a promising source of physiological biomarkers accompanying chronic pain. Several studies in patients with chronic neuropathic pain have reported alterations in central pain processing, manifested as slowed EEG rhythmicity and increased EEG power in the brain's resting state. We aimed to investigate novel potential markers of chronic pain in the resting state EEG of patients with chronic pancreatitis.

Participants: Resting state EEG data from 16 patients with persistent abdominal pain due to chronic pancreatitis (CP) were compared to data from healthy controls matched for age, sex and education.

Methods: The peak alpha frequency (PAF) and power amplitude in the alpha band $(7.5-13 \mathrm{~Hz})$ were compared between groups in four regions of interest (frontal, central, parietal, and occipital) and were correlated with pain duration.

Results: The average PAF was lowered in CP patients compared with that in healthy controls, observed as a statistically significant between-group effect (mean 9.9 versus $9.5 \mathrm{~Hz} ; P=0.049$ ). Exploratory post hoc analysis of average PAF per region of interest revealed a significant difference, particularly in the parietal and occipital regions. In addition, we observed a significant correlation between pain duration and PAF and showed increased shifts in PAF with longer pain durations. No significant group differences were found in peak power amplitudes.

Conclusion: CP pain is associated with alterations in spontaneous brain activity, observed as a shift toward lower PAF. This shift correlates with the duration of pain, which demonstrates that PAF has the potential to be a clinically feasible biomarker for chronic pain. These findings could be helpful for assisting diagnosis, establishing optimal treatment, and studying efficacy of new therapeutic agents in chronic pain patients.

Keywords: chronic pain, neuropathic pain, chronic pancreatitis, electroencephalography, EEG, alpha oscillations, peak frequency

\section{Introduction}

Diagnosis and treatment of chronic pain is challenging because by definition, pain is a subjective experience and can be measured only by self-report. ${ }^{1}$ Identification of physiological pain biomarkers for 1) disease severity, 2) disease progression, 3) disease prognosis, and 4) treatment effects, including indication and responder identification, could help us to improve pain diagnostics and treatment. Increasing evidence supports the idea that chronic pain can be understood not only as an altered perceptual state, but also as a consequence of alterations in peripheral and central neuronal processing.

Electroencephalography (EEG) can be a useful method to detect such alterations in central pain processing. ${ }^{2-4}$ The resting state EEG with eyes closed is dominated by oscillations in the alpha band $(7.5-13 \mathrm{~Hz})$, which are widely distributed in the cerebral 
cortex and more prominent in the parietal and occipital regions. Resting EEG is commonly analyzed by transforming data from the time domain to the frequency domain. The peak alpha frequency (PAF) is a measure derived from that analysis, and is defined by two parameters: 1) the frequency at which it occurs on the frequency axis; and 2) its amplitude on the power-density axis.

Sarnthein et $\mathrm{al}^{2}$ observed increased power amplitude differences in the alpha band, and a shift toward lower frequencies of the dominant peak in patients with mixed neurogenic pain syndromes. These results are supported by other resting state EEG studies investigating alterations in central pain processing in various chronic pain states. ${ }^{3,5-7}$ Similar alterations in EEG activity were reported in patients with chronic pancreatitis, observed as an increase in power amplitude in the theta and alpha frequency bands. ${ }^{4,8}$ However, PAF and its relation to clinical pain parameters were not investigated in these studies.

Chronic pancreatitis (CP) is a disease characterized by inflammation and progressive destruction of the pancreatic gland, which results in irreversible morphologic changes that typically cause pain and/or exocrine and endocrine insufficiency. ${ }^{9}$ The most important symptom of CP is abdominal pain, present in $80 \%-90 \%$ of patients during the time course of the disease. ${ }^{10}$ Pancreatic pain is typically intense, long-lasting, and difficult to treat. Alterations in pancreatic nerves, including an increase in number and diameter of nerve fibers and an increased number of neurotransmitters, ${ }^{11,12}$ as well as alterations in central pain processing, including supraspinal sensitization, somatotopic reorganization, and pro-nociceptive pain modulation, have been proposed as possible mechanisms underlying chronic pain in $\mathrm{CP} .{ }^{13-15}$ Altered central pain processing was demonstrated in a previous study in CP patients using quantitative sensory testing, manifested as a widespread hyperalgesia, ie, an increased pain sensitivity, ${ }^{16}$ in distant, nondamaged tissues. This can be interpreted as a sign of spinal, supraspinal (cortical), or combined sensitization. ${ }^{17}$ These observations support the role of central neuronal plasticity in the pain accompanying CP. If that is correct, therapies exclusively directed at the pancreas as the nociceptive source are unlikely to be effective in achieving pain relief. Therefore, patients who might benefit from a treatment targeting central pain mechanisms need to be identified.

In the current study, we aim to investigate the brain's resting state activity within the alpha frequency band in patients with chronic pain resulting from $\mathrm{CP}: 1)$ to research novel potential EEG biomarkers; 2) to investigate biomarker scalp localization; 3 ) to study effects of disease progression on biomarkers; and 4) to address the clinical usefulness of EEG biomarkers for CP pain.

\section{Methods Subjects}

Sixteen patients with persistent abdominal pain resulting from CP were selected at random from those with CP seen at the outpatient clinic of the Radboud University Nijmegen Medical Centre, Nijmegen, the Netherlands. CP was diagnosed based on medical history, laboratory tests, and radiological findings according to the Marseille and Cambridge Classification System. ${ }^{18}$ All patients had typical pancreatic pain, which is characterized as severe, dull epigastric pain, eventually radiating to the back. Intake of analgesics including opioids and centrally acting medication was permitted. Patients with present alcohol use were excluded. The healthy controls (HC) group comprised 16 healthy participants matched by age, sex, and years of education to the CP group. Previous studies suggest that this is an appropriate sample size to investigate the resting state EEG., ${ }^{2,6,19}$

Medical-ethical approval was obtained for the measurements in HCs (Committee on Research involving Human Subjects, Region Arnhem-Nijmegen Number 2002/008). All patients were referred by their physician in charge for neuropsychological/neurophysiological testing as part of their medical follow-up. The neurophysiologic testing results have already been published and revealed a decline in cognitive performance in the $\mathrm{CP}$ group. ${ }^{20}$ Both patients and healthy participants gave written informed consent to use the data for scientific purposes.

\section{EEG recording}

EEG data were collected according to a standardized protocol using a Quickcap (NuAmps, Compumedics Neuroscan, Singen, Germany) with 26 scalp electrodes located according to the International 10-20 system (Fp1, Fp2, F7, F3, Fz, F4, F8, FC3, FCz, FC4, T3, C3, Cz, C4, T4, CP3, CPz, CP4, T5, P3, Pz, P4, T6, O1, Oz, O2). ${ }^{21}$ Electrooculogram data were recorded from electrodes above and below the left eye and lateral to the outer canthi of each eye. Additional physiological data were obtained from the orbicularis oculus and the masseter muscles. Data were recorded at a sampling rate of $500 \mathrm{~Hz}$ and offline referenced to the mean of the signals recorded at the mastoids. The ground electrode was placed at the Fpz location. Electrode impedance was kept below 5 $\mathrm{k} \Omega$ for all electrodes.

The spontaneous EEG or resting EEG was recorded during eyes closed and eyes open. Each recording lasted 2 minutes. 
All results presented in this study refer to the eyes-closed condition to avoid artifacts; alpha activity is typically present during this condition. During the recording in eyes-closed position, participants were seated in a comfortable chair and were asked to close their eyes and relax. No further task was given.

\section{EEG analysis}

Brain Vision Analyzer 2.0 software (Brain Products $\mathrm{GmbH}$, Gilching, Germany) was used for EEG analysis. EEG data were band-pass filtered (1-120 Hz; phase shift-free Butterworth filters), and corrected for ocular artifacts according to the Gratton and Coles algorithm. ${ }^{22}$ Each EEG recording was segmented into 12 epochs of 10 seconds each. Subsequently, epochs were inspected for artifacts and rejected for further analysis if data exceeded an amplitude of $200 \mu \mathrm{V}$ or exceeded the maximal allowed voltage step of $50 \mu \mathrm{V}$. This resulted in $1.7 \%$ rejection of all epochs, mainly those concerning temporal electrodes. The power amplitudes of the EEG frequencies were computed using a fast Fourier transformation. To this end, epochs were multiplied by a Hanning window (10\%) and Fourier-transformed; spectral distributions were averaged across all epochs for each participant and electrode separately.

\section{Data analysis and statistics}

Grand average power spectra were computed by averaging all scalp electrodes for each participant. These grand averages were averaged per group to obtain the overall power. Peak power amplitudes were determined as the maximum value between $7.5-13 \mathrm{~Hz}$ within empirically defined regions of interest (ROIs). Positively skewed peak power amplitudes were log-transformed to normalize the data. A lack of lateralization, as shown in topographical distribution plots, provides the opportunity to average individual electrodes in the ROIs to obtain a more stable, but targeted, analysis. Hence, four horizontally arranged ROIs were designated: the frontal (Fp1, Fp2, F3, Fz, F4), central (FC3, FCz, FC4, C3, $\mathrm{Cz}, \mathrm{C} 4)$, parietal (CP3, CPz, CP4, P3, Pz, P4), and occipital (O1, Oz, O2) ROIs.

Different methods can be used to quantify the variation of spectral distribution within the alpha range. ${ }^{23}$ First, PAF can be measured by calculating the frequency with the highest magnitude within the alpha range. Second, the center of gravity, rather than peak, can be measured. This gravity method has been used as a different, and possibly more stable, measure of spectral distribution than the peak method. ${ }^{23,24}$ In particular, if there are multiple peaks in the alpha range, the gravity method appears the more adequate estimate of the PAF. ${ }^{23}$ In the current study, a few participants demonstrated low-voltage EEG without clear peaks within the alpha band. The center of gravity method was assumed to be most appropriate, because this method enables analysis of the entire dataset without excluding low-voltage EEG subjects from analysis. All participants demonstrated at least some peak within the $7.5-13 \mathrm{~Hz}$ range, assumed as the alpha frequency band and were included for further analyses. The PAF is the weighted sum of spectral estimates, divided by alpha power, as calculated by the following equation: ${ }^{25}$

$$
\mathrm{PAF}=\Sigma\left(\mathrm{a}_{\mathrm{f}} \times \mathrm{f}\right) / \Sigma \mathrm{a}_{\mathrm{f}}
$$

$\mathrm{a}_{\mathrm{f}}=$ Amplitude of frequency $\mathrm{f}$

$\mathrm{f}=$ Frequencies within $7.5-13 \mathrm{~Hz}$ range (per $0.1 \mathrm{~Hz}$ ).

For statistical analysis, we used SPSS software for Windows version 16.0 (IBM Corporation, Armonk, NY, USA). All variables were visually inspected and the KolmogorovSmirnoff Test was applied to test data distributions. A $t$-test for independent samples was applied on normally distributed data; otherwise a non-parametric Mann-Whitney $U$ test was used. A General Linear Model (GLM; StatSoft, Tulsa, OK, USA) repeated measures analysis of variance (RM-ANOVA) was used to test whether there were statistically significant differences regarding PAF and peak power amplitudes between $\mathrm{CP}$ patients and $\mathrm{HCs}$ with respect to the ROI (frontal, central, parietal and occipital). Our dependent variable, the PAF, was normally distributed, allowing parametric testing. Mauchly's test indicated that the assumption of sphericity had been violated. Therefore, degrees of freedom were corrected using Greenhouse-Geisser estimation. Post hoc analyses included exploratory pair-wise testing of each region of interest separately, using two-sided unpaired $t$-tests. A GLM repeated-measures ANOVA was used to test whether there were significant differences between opioid and nonopioid users and between the different etiologies of $\mathrm{CP}$, with respect to the ROI. In addition, pain duration was correlated with EEG parameters using the nonparametric Spearman test. Controls did not have pain and were allocated zero scores on pain duration, and were included in this analysis. In all tests, the significance level was set at $P<0.05$.

\section{Results}

\section{Research population}

The CP patients had a mean pain duration of 5.4 years; eight patients had a history of alcohol abuse and nine patients used opioid medication for pain relief (Table 1). Matched controls 
Table I Demographic and clinical characteristics of individual patients with chronic pancreatitis

\begin{tabular}{|c|c|c|c|c|c|c|}
\hline No & $\begin{array}{l}\text { Age } \\
\text { (years) }\end{array}$ & Sex & Etiology & $\begin{array}{l}\text { Pain } \\
\text { (years) }\end{array}$ & Opioids & Other drugs \\
\hline I & 28 & $F$ & Hereditary & 6 & MS-Contin ${ }^{\circledast}$ & PPI \\
\hline 2 & 50 & M & Idiopathic & 5 & - & PPI \\
\hline 3 & 57 & $\mathrm{~F}$ & Alcohol abuse & 10 & Durogesic & - \\
\hline 4 & 40 & $M$ & Alcohol abuse & 6 & Morphine & PM; NSAID \\
\hline 5 & 51 & $M$ & Alcohol abuse & 6 & Temgesic & - \\
\hline 6 & 54 & $M$ & Alcohol abuse & 8 & Morphine & $A D$ \\
\hline 7 & 58 & M & Alcohol abuse & 4 & Tramadol & PM; AE \\
\hline 8 & 39 & $\mathrm{~F}$ & Idiopathic & 10 & Durogesic/pethidine & - \\
\hline 9 & 46 & $\mathrm{~F}$ & Idiopathic & 2 & Oxycontin & - \\
\hline 10 & 72 & $M$ & Idiopathic & 6 & - & PPI; NSAID \\
\hline II & 50 & $M$ & Alcohol abuse & 10 & - & PM \\
\hline 12 & 48 & $M$ & Biliary & 4 & Oxycontin & $A E ; B Z ; P M$ \\
\hline 13 & 56 & $M$ & Alcohol abuse & 2 & - & PM; PPI \\
\hline 14 & 24 & $\mathrm{~F}$ & Idiopathic & 2 & - & - \\
\hline 15 & 52 & $\mathrm{~F}$ & Alcohol abuse & 5 & - & AE; BZ; PM; Li \\
\hline 16 & 59 & $M$ & Idiopathic & I & - & PPI \\
\hline Mean (SD) & & & & $5,4(2,9)$ & & \\
\hline
\end{tabular}

Notes: Relevant drugs include antiepileptics (AE), benzodiazepines (BZ), antidepressants (AD), lithium (Li), non-steroidal anti-inflammatory drug (NSAID), paracetamol (PM) and proton pump inhibitors (PPI).

Abbreviations: F, female; M, male; SD, standard deviation; MS, morphine sulfate.

did not use centrally acting medication and all were pain free, expressed as zero scores on pain duration. No differences were observed between the $\mathrm{CP}$ and $\mathrm{HC}$ group with respect to age, sex, and years of education (Table 2).

\section{Grand average power spectra}

Absolute values of grand average power spectra amplitudes within the alpha band are summarized for CP patients and $\mathrm{HC}$ in Figure 1. No significant group differences were found in the logarithmically transformed peak power amplitudes. The corresponding PAF was significantly shifted toward lower EEG frequencies in the CP group compared with the HC group (mean \pm SD: $9.9 \pm 0.4$ versus $9.5 \pm 0.5 \mathrm{~Hz}$; $95 \%$ confidence interval $[\mathrm{CI}]$ of mean difference $=-0.68$ to $-0.01 \mathrm{~Hz} ; P<0.05)$. Moreover, pain duration was significantly correlated with the grand average PAF $(\mathrm{r}=-0.379$; $P=0.032$ ), showing increased reductions in PAF with longer pain durations (Figure 2).

Table 2 Demographic and clinical characteristics of healthy controls and chronic pancreatitis patients

\begin{tabular}{llll}
\hline & HC & CP & P-value \\
\hline $\mathrm{N}$ & 16 & 16 & \\
Male/female & $10 / 6$ & $10 / 6$ & $\mathrm{NS}$ \\
$\begin{array}{l}\text { Mean (SD) } \\
\text { age (years) }\end{array}$ & $48.0(1 \mathrm{I} .27)$ & $49.5(\mathrm{II} .9 \mathrm{I})$ & $\mathrm{NS}$ \\
$\begin{array}{l}\text { Mean (SD) } \\
\text { education (years) }\end{array}$ & $11.9(2.86)$ & $11.8(3.09)$ & $\mathrm{NS}$ \\
\hline
\end{tabular}

Abbreviations: $\mathrm{HC}$, healthy controls; $\mathrm{CP}$, chronic pancreatitis patients; NS, not significant; $\mathrm{N}$, number; $\mathrm{SD}$, standard deviation.

\section{Topographical power distributions}

Differences in grand average power spectra between both groups were restricted to the frequency range between 7.5 and $10 \mathrm{~Hz}$ (Figure 1). Thus, we restricted the topographical analysis of EEG power to this part of the alpha band (Figure 3A-F). The topographical distribution plots showed maximum EEG alpha power in both groups, as well as maximum group differences, to be situated in the parietal and occipital regions.

\section{Power spectra}

The average power frequency distributions were plotted separately per ROI in Figure 4A-D. This Figure suggests increased peak power amplitudes in CP patients compared with the HC group in each of the ROIs, particularly parietal and occipital. However, logarithmically transformed peak power amplitudes did not differ significantly in any of the ROIs (Table 3).

\section{Peak alpha frequency}

The mean PAF for each of the ROIs is shown in Figure 5. A statistically significant between-group effect was observed regarding the $\mathrm{PAF}$ in $\mathrm{CP}$ patients compared with $\mathrm{HC}(F=4.20$; $P=0.049)$. Within-group testing revealed a statistically significant difference among the ROIs $(F=11.62 ; P<0.001)$. No significant interaction was observed between the effects of group and ROIs on the $\mathrm{PAF}(F=2.785 ; P=0.085)$. Exploratory post hoc testing resulted in significant differences between 


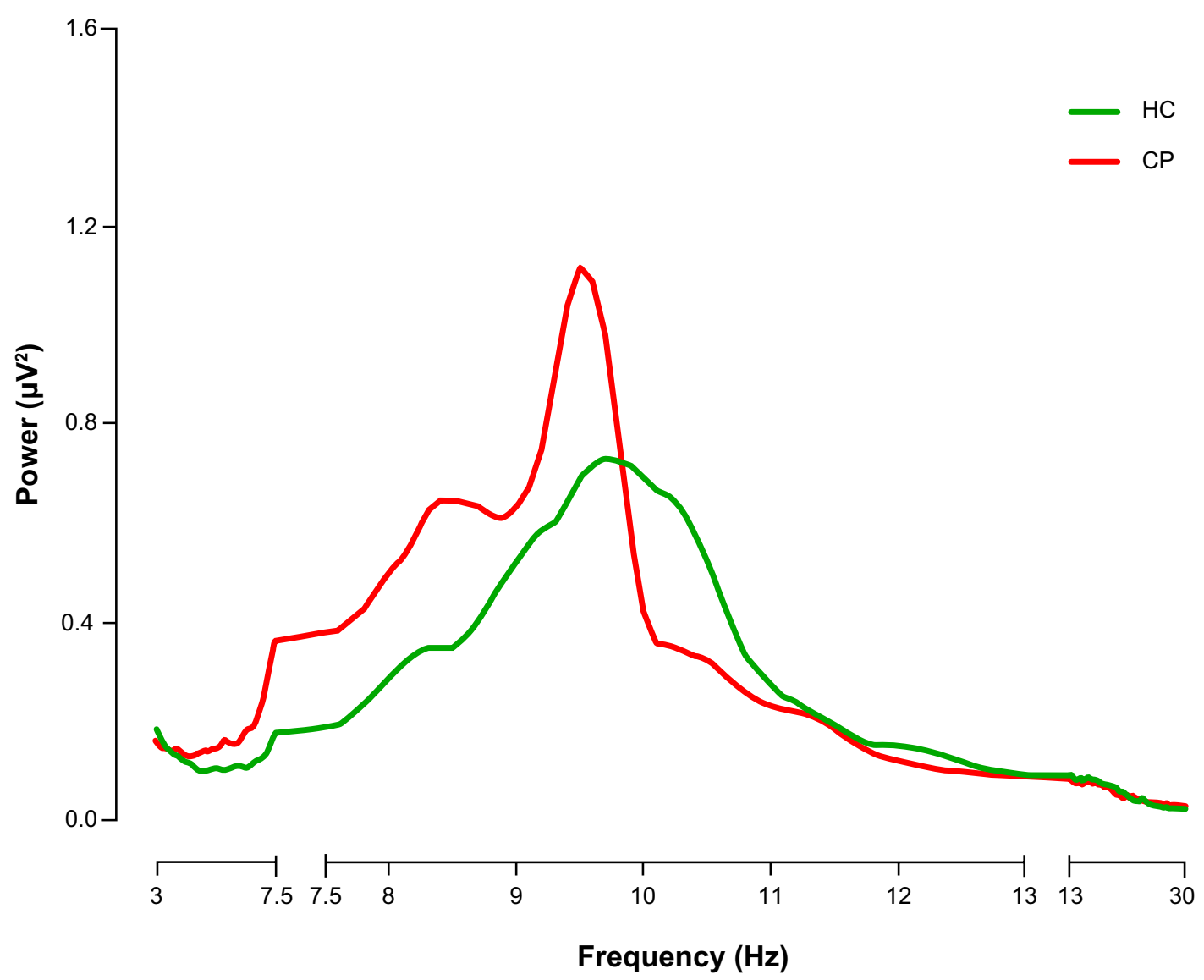

Figure I Grand average frequency power distributions averaged across all channels in patients with chronic pancreatitis (CP) compared to healthy controls (HC). Notes: This figure shows a shift toward lower frequencies and an increased amplitude in CP patients compared with $\mathrm{HC}$.

patients and controls regarding the PAF in the parietal and occipital ROIs (Table 3).

The mean PAF in CP patients using opioid medication and nonopioid medication was similar: $9.5 \pm 0.5 \mathrm{~Hz}$ and

Table 3 Peak alpha frequency (PAF) and logarithmized peak power in healthy controls $(\mathrm{HC})$ and chronic pancreatitis patients (CP)

\begin{tabular}{|c|c|c|c|c|c|}
\hline & \multicolumn{4}{|c|}{ Mean (SD) } & \multirow[t]{2}{*}{$P$} \\
\hline & $\mathrm{HC}$ & & CP & & \\
\hline \multicolumn{6}{|l|}{ Frontal ROI } \\
\hline PAF & 9.7 & $(0.50)$ & 9.4 & $(0.46)$ & 0.190 \\
\hline Logarithmized peak power & -0.39 & $(0.98)$ & -0.19 & $(1.09)$ & 0.586 \\
\hline \multicolumn{6}{|l|}{ Central ROI } \\
\hline PAF & 9.8 & $(0.42)$ & 9.5 & $(0.49)$ & 0.091 \\
\hline Logarithmized peak power & -0.31 & $(1.08)$ & -0.04 & $(0.97)$ & 0.462 \\
\hline \multicolumn{6}{|l|}{ Parietal ROI } \\
\hline PAF & 9.9 & $(0.4 I)$ & 9.6 & $(0.50)$ & $0.037 *$ \\
\hline Logarithmized peak power & 0.21 & $(0.97)$ & 0.31 & $(1.34)$ & $0.81 \mathrm{I}$ \\
\hline \multicolumn{6}{|l|}{ Occipital ROI } \\
\hline PAF & 10.0 & $(0.47)$ & 9.6 & $(0.59)$ & $0.019 *$ \\
\hline Logarithmized peak power & -0.24 & (I.59) & 0.32 & (I.59) & 0.332 \\
\hline
\end{tabular}

Note: $* P<0.05$.

Abbreviations: $\mathrm{ROI}$, regions of interest; SD, standard deviation.
$9.5 \pm 0.5 \mathrm{~Hz}$, respectively. Opioid use as between-group factor in the RM-ANOVA indicated no significant differences regarding $\mathrm{PAF}(F=0.015 ; P=0.904)$ or peak power amplitudes $(F=1.593 ; P=0.228)$. Opioid use as covariate did not modify the main between-group effect. Subgroups of patients with or without alcohol abuse in history did not show significant differences regarding $\operatorname{PAF}(F=0.063 ; P=0.806)$ or peak power amplitudes $(F=1.984 ; P=0.181)$.

\section{Discussion}

We observed a significant shift toward lower frequencies in patients with CP compared with healthy controls, recorded as a decrease in PAF over all scalp electrodes. These results are consistent with other studies investigating the brain's default state in chronic pain patients, including patients with $\mathrm{CP}$, reported as a slowing of EEG oscillations..$^{2-5,7}$ Exploratory post hoc analysis of average PAF per ROI reveals a significant difference, particularly in parietal and occipital regions. Furthermore, the present study shows that longer pain durations are associated with greater declines in PAF, indicating that PAF might be a marker for disease progression. 


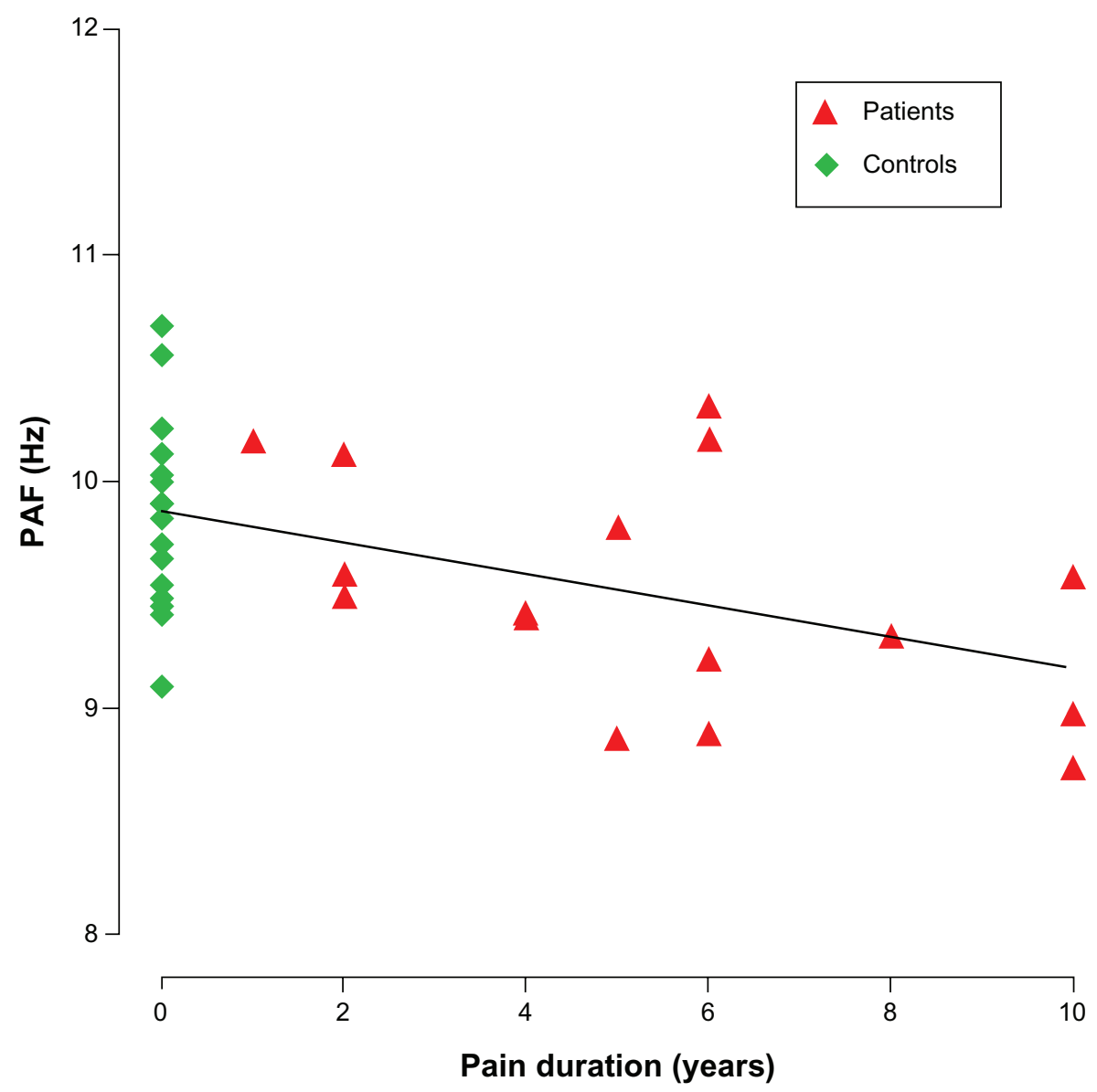

Figure 2 Individual pain durations and grand average peak alpha frequencies of both chronic pancreatitis patients and healthy controls (HC).

Notes: $\mathrm{HC}$ were all pain free, expressed as zero scores on pain duration. A significant correlation was found $(r=-0.379 ; P=0.032)$, indicating that an increase in pain duration is correlated with an increased shift of PAF.

Abbreviation: PAF, peak alpha frequency.

\section{Alpha oscillations in the resting state EEG}

Continuous EEG is dominated by alpha band oscillations $(7.5-13 \mathrm{~Hz})$, which are widely distributed in the cerebral cortex and recorded with larger amplitudes over posterior regions with the eyes closed. ${ }^{25}$ The exact role of alpha oscillations remains unclear, but several factors have been identified affecting the alpha activity in some way. The PAF, a primary measure of alpha activity, starts to decline with increasing $\operatorname{age}^{26}$ and is known to increase with cognitive processing, attentional demand, and arousal. ${ }^{27}$ Several studies have found PAF to be a stable measure, showing a high intra-individual stability. ${ }^{28,29}$

\section{Spontaneous alpha oscillations in chronic pain}

Multiple studies report that phasic, as well as tonic, painful stimuli suppress spontaneous oscillations over the cortex in healthy participants, ${ }^{19,30}$ but only a few studies have investigated the brain default state in patients with chronic pain. Sarnthein et $\mathrm{al}^{2}$ reported an increased EEG power and a slowed dominant peak frequency in patients with severe neuropathic pain of various origins. Maximal differences appeared in the 7-9 Hz band in all electrodes. These results were explained by the concept of thalamocortical dysrhythmia (TCD), which is proposed as a general mechanism to explain the generation of neuropathic pain and other neurological symptoms. ${ }^{2,3,31} \mathrm{TCD}$ is based on diminished excitatory or increased inhibitory input of neurons in the thalamus, resulting in the presence of a persistent low-frequency, thalamocortical resonance during the awake state. ${ }^{3}$ This mechanism has been supported by the finding that therapeutic surgical lesions in the thalamus resulted in normalization of EEG activity as well as providing pain relief. ${ }^{2}$

Two studies in patients with neuropathic pain following spinal cord injury (SCI) support the TCD theory. In both studies, peak frequency was shifted towards lower frequencies in SCI patients with pain as compared with SCI patients without pain. In contrast, no differences were observed in power amplitudes. ${ }^{5,7}$ Interestingly, this is not the case in 

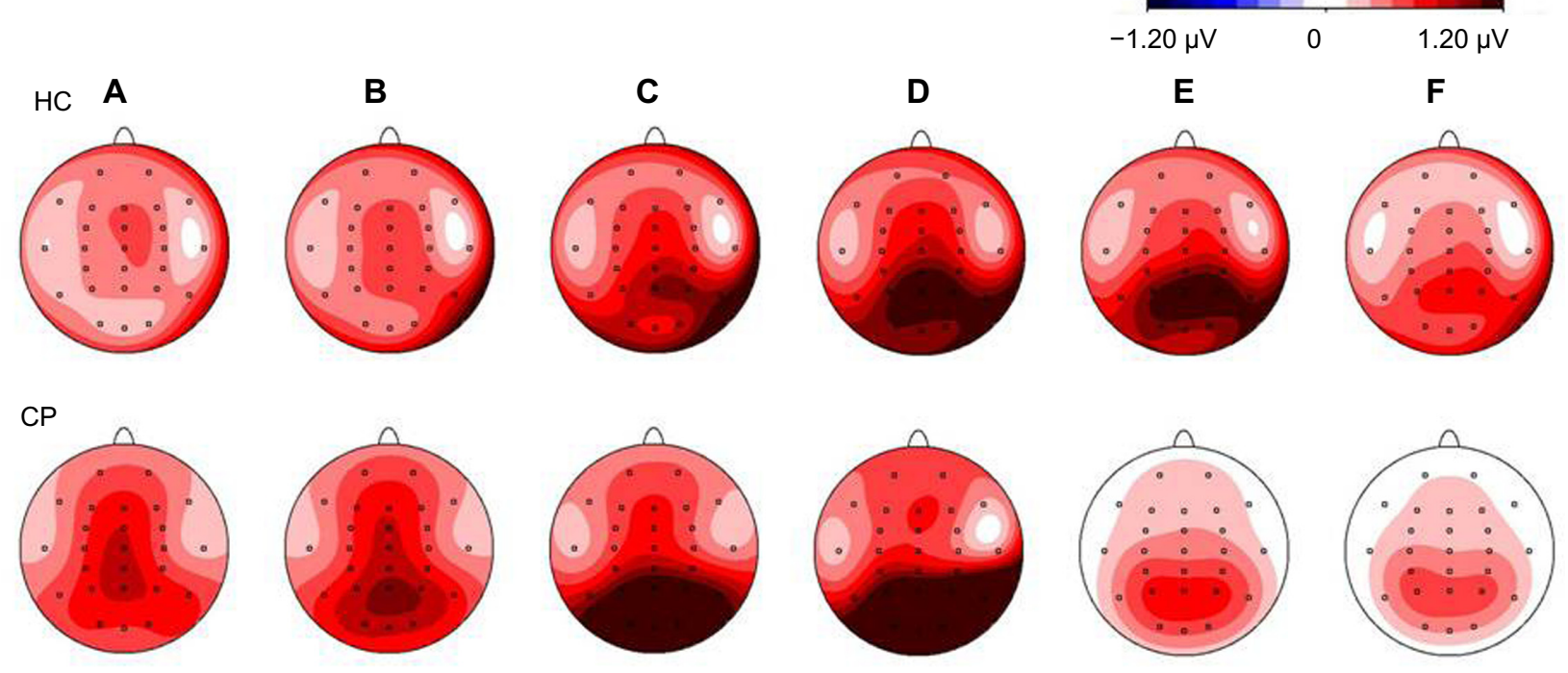

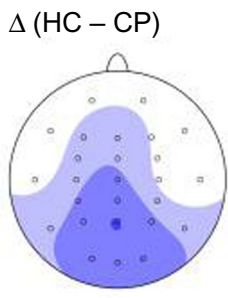

A; $7.5-8.0 \mathrm{~Hz}$

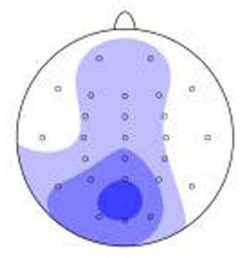

B; $8.0-8.5 \mathrm{~Hz}$

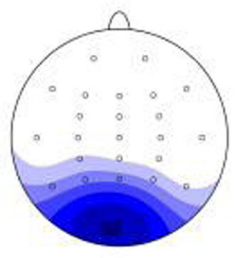

C; $8.5-9.0 \mathrm{~Hz}$

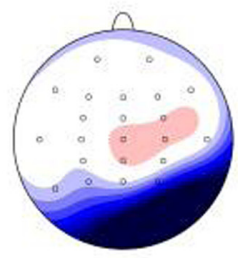

D; $9.0-9.5 \mathrm{~Hz}$

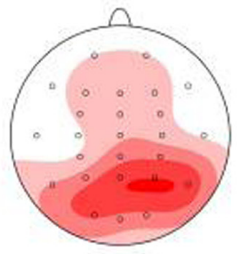

E; $9.5-10.0 \mathrm{~Hz}$

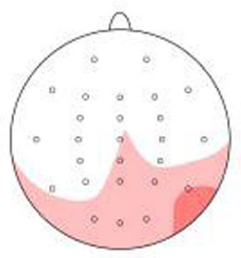

F; $10.0-10.5 \mathrm{~Hz}$

Figure 3 Average topographical power distributions of the resting electroencephalogram.

Notes: The average topographical distributions of electroencephalography power showed the maximum amplitudes in the parietal-occipital regions in both chronic pancreatitis $(\mathrm{CP})$ patients and healthy controls $(\mathrm{HC})$. Scalp distributions are shown for frequency spectra within the alpha band.

patients with chronic low back pain, who did not demonstrate any statistically significant TCD effect, according to a study by Schmidt et al. ${ }^{32}$ Only in a subsample of patients showing evidence of root damage was a trend for significant effect observed. The authors of that study suggest that only patients with severe pain or neuropathic pain develop the typical TCD pattern. This may be, as stated earlier, because pancreatic pain is typically intense and long lasting, and also because pancreatic pain may be of neuropathic origin. ${ }^{8,33}$

A previous study in CP patients with pain showed slowing of EEG rhythms based on increased normalized power amplitudes in the lower frequency bands, including the alpha band. ${ }^{4}$ The present study confirms those results in a similar population of $\mathrm{CP}$ patients. It extends those results to additional EEG parameters, better quantifying alpha-slowing using PAF, and establishes a relation to clinically relevant factors such as pain duration. Interestingly, based on our PAF, maximum differences between groups were located over the posterior regions, whereas Olesen et al ${ }^{4}$ reported that mainly the frontal electrodes contributed to the difference based on normalized amplitude strengths. However, both studies observed slowing of EEG rhythmicity, which suggests that pancreatic pain originates from a disturbance in thalamocortical rhythmia.

\section{Toward a biomarker for chronic pain}

A simple self-evaluation of pain is not sufficient to provide insight into underlying mechanisms because multiple factors potentially affect the pain experience. Therefore, development of a physiological measure reflecting underlying pain mechanisms is desirable. First, it may improve pain diagnostics through addition of a mechanism-oriented parameter that reflects central neuronal involvement in pain genesis and maintenance. Second, it may improve pain treatment by identification of patients who may benefit from a treatment targeting central pain mechanisms. Graversen et $\mathrm{a}^{34}$ have shown that quantitative pharmaco-EEG can be used to monitor the central analgesic mechanisms of pregabalin, and they suggest that this approach may be used to predict effect of treatment leading to pharmaco-diagnostic testing. 

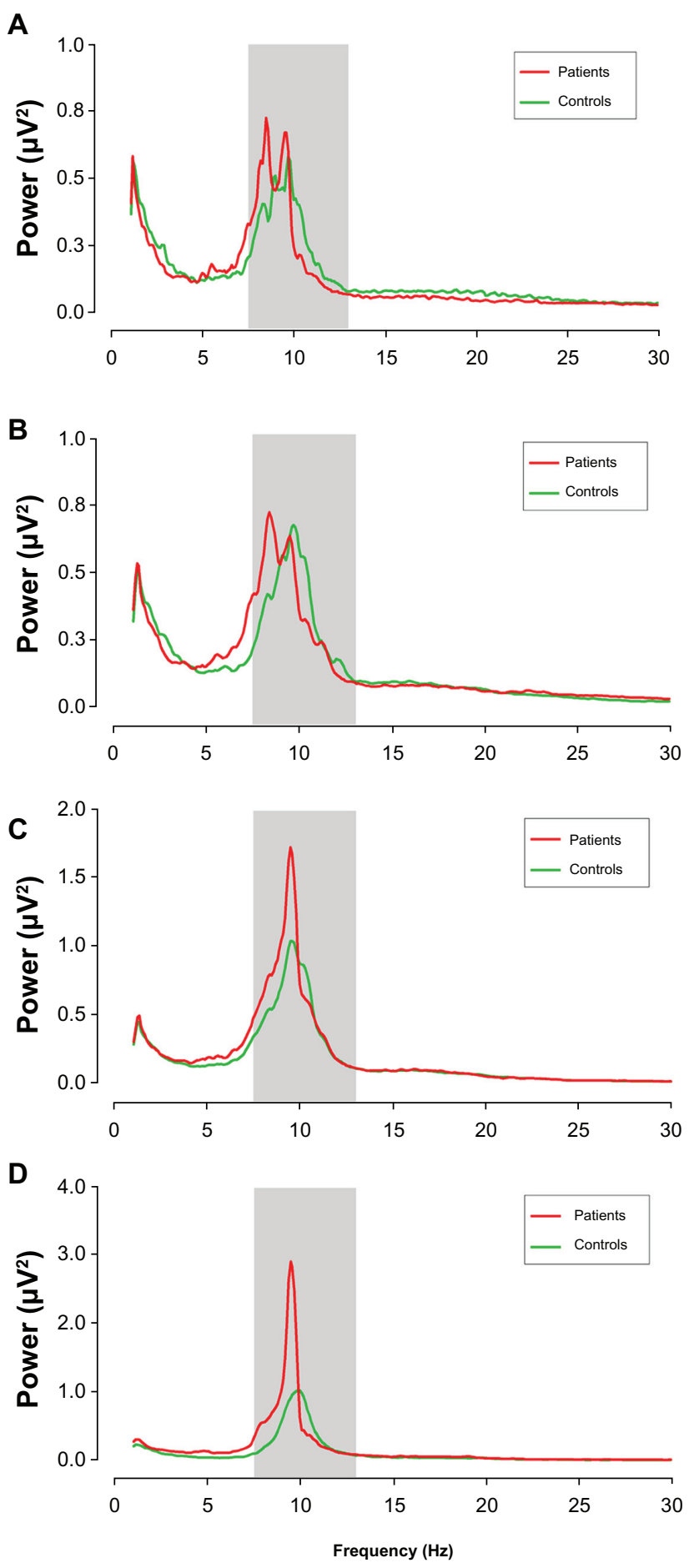

Figure 4 Averaged power distributions within the frontal (A), central (B), parietal (C), and occipital (D) regions of interest in chronic pancreatitis patients compared to healthy controls.

Note: The grey square represents the area within the $\alpha$-band.

\section{Clinical applications of EEG}

Beside the fact that EEG measures different phenomena regarding brain function than functional MRI (fMRI) or positron emission tomography (PET), EEG has several advantages: 1) PET and fMRI are based on the measurement

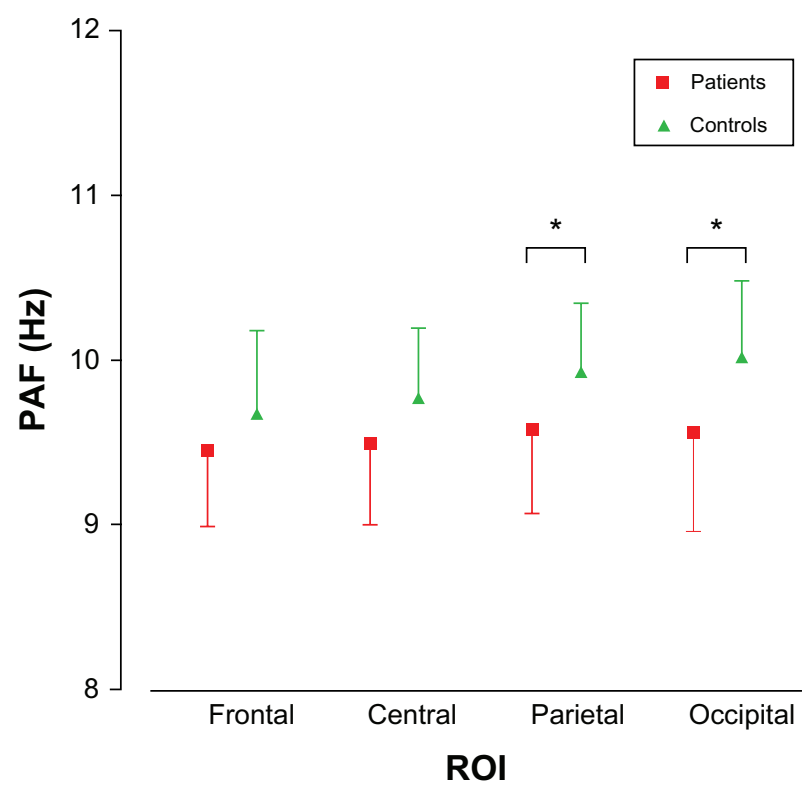

Figure 5 Peak alpha frequency in four regions of interest shown for patients with chronic pancreatitis compared with healthy controls.

Notes: Red squares correspond to mean PAF in patients, green triangles correspond to mean PAF in controls, and short lines represent corresponding standard deviations. Asteriks indicate significant differences.

Abbreviations: ROI, region of interest; PAF, peak alpha frequency.

of secondary metabolic changes in brain tissue, but not of primary electrical effects of neural excitation; 2) EEG equipment costs significantly less than neuro-imaging equipment; and 3) EEG equipment, including electrodes, a signal amplifier, and a computer with EEG software, is portable and easy to apply. This enables us to record the EEG near the patient's bed. Conversely, it usually takes a long time to apply numerous electrodes at the scalp; therefore, it would be desirable to reduce the number of required electrodes in clinical practice. But which electrodes are superfluous? Our study showed the maximum alpha-band oscillations in both groups to be located in the parietal and occipital regions. More importantly, differences between groups in PAF, the only discriminative parameter observed in our study, were located over the same posterior regions. This suggests that PAF is best measured in the parietal and occipital regions of the scalp for chronic pain diagnostics.

\section{Methodological considerations}

Future research should concentrate on limitations within the current study. First, we did not collect pain scores during or just preceding the measurement or average pain scores over the past few months. Therefore, it was not possible for us to correlate pain intensities with EEG parameters. Second, we made a comparison of $\mathrm{CP}$ patients with pain versus healthy participants to study the differential influence of chronic pain. 
We recruited a homogeneous group of patients, all suffering from persistent visceral pain resulting from diagnosed CP. Although these patients were homogeneous with respect to the cause of pain, it is difficult to ascribe the observed changes in the resting EEG to just one underlying cause. Variations in pain duration, as well as differences in etiology (eg, history of alcoholism), comorbidity (eg, exocrine and/or endocrine failure), surgical treatment history, and actual medication use may be contributing factors. Thus, it might be interesting to investigate the influence of these factors based on a third group of CP patients without pain. However, it will be challenging to find $\mathrm{CP}$ patients having no pain and matched by age, level of education, and medication intake, which are evident factors effecting the resting EEG.

Centrally acting medication might influence the brain's resting-state activity. Many patients with $\mathrm{CP}$ use analgesics, including opioids, for pain treatment. This presents an ethical dilemma, as patients could potentially face severe pain if their medication were discontinued. In our study, more than half of the patients used opioids at the time of measurements. A comparison between subgroups of patients with and without opioids did not reveal any significant difference. Hence, the slowed PAF observed in our study is unlikely to have been caused by centrally acting medication.

\section{Conclusion}

The present study shows a shift of the alpha peak towards lower frequencies in $\mathrm{CP}$ patients with chronic pain compared with healthy controls. This shift correlates with the duration of pain, which demonstrates that PAF deserves further study regarding its potential as a clinically useful biomarker for chronic pain. The subdivision in four ROIs showed that this biomarker is best measured in the parietooccipital regions of the scalp, which reduces the number of electrodes necessary; this is of benefit for clinical practice. Accordingly, this method appears promising in supporting diagnosis and prognosis, establishing optimal treatment, and studying efficacy of new therapeutic agents in chronic pain patients.

\section{Acknowledgments}

The study was investigator-initiated and financially supported by the Department of Surgery of the Radboud University Nijmegen Medical Centre, Nijmegen, the Netherlands. The results have been presented at the International Association for the Study of Pain (IASP) 14th World Congress on Pain; August 27-31, 2012; Milan, Italy.

\section{Disclosure}

The authors have no conflicts of interest to declare.

\section{References}

1. Davis KD, Racine E, Collett B. Neuroethical issues related to the use of brain imaging: Can we and should we use brain imaging as a biomarker to diagnose chronic pain? Pain. 2012;153(8):1555-1559.

2. Sarnthein J, Stern J, Aufenberg C, Rousson V, Jeanmonod D. Increased EEG power and slowed dominant frequency in patients with neurogenic pain. Brain. 2006;129(Pt 1):55-64.

3. Llinás RR, Ribary U, Jeanmonod D, Kronberg E, Mitra PP. Thalamocortical dysrhythmia: A neurological and neuropsychiatric syndrome characterized by magnetoencephalography. Proc Natl Acad Sci U S A. 1999;96(26):15222-15227.

4. Olesen SS, Hansen TM, Graversen C, Steimle K, WilderSmith $\mathrm{OH}$, Drewes AM. Slowed EEG rhythmicity in patients with chronic pancreatitis: evidence of abnormal cerebral pain processing? Eur J of Gastroenterol Hepatol. 2011;23(5):418-424.

5. Boord P, Siddall PJ, Tran Y, Herbert D, Middleton J, Craig A Electroencephalographic slowing and reduced reactivity in neuropathic pain following spinal cord injury. Spinal Cord. 2008;46(2):118-123.

6. Sarnthein J, Jeanmonod D. High thalamocortical theta coherence in patients with neurogenic pain. Neuroimage. 2008;39(4):1910-1917.

7. Wydenkeller S, Maurizio S, Dietz V, Halder P. Neuropathic pain in spinal cord injury: significance of clinical and electrophysiological measures. European J Neurosci. 2009;30(1):91-99.

8. Drewes AM, Gratkowski M, Sami SA, Dimcevski G, Funch-Jensen P, Arendt-Nielsen L. Is the pain in chronic pancreatitis of neuropathic origin? Support from EEG studies during experimental pain. World $J$ Gastroenterol. 2008;14(25):4020-4027.

9. Schneider A, Lohr JM, Singer MV. The M-ANNHEIM classification of chronic pancreatitis: introduction of a unifying classification system based on a review of previous classifications of the disease. J Gastroenterol. 2007;42(2):101-119.

10. Andren-Sandberg A, Hoem D, Gislason H. Pain management in chronic pancreatitis. Eur J Gastroenterol Hepatol. 2002;14(9):957-970.

11. Di Sebastiano P, di Mola FF, Bockman DE, Friess H, Buchler MW. Chronic pancreatitis: the perspective of pain generation by neuroimmune interaction. Gut. 2003;52(6):907-911.

12. Friess H, Shrikhande S, Shrikhande M, et al. Neural alterations in surgical stage chronic pancreatitis are independent of the underlying aetiology. Gut. 2002;50(5):682-686.

13. Vanegas H, Schaible HG. Descending control of persistent pain: inhibitory or facilitatory? Brain Res Brain Res Rev. 2004;46(3):295-309.

14. Porreca F, Ossipov MH, Gebhart GF. Chronic pain and medullary descending facilitation. Trends Neurosci. 2002;25(6):319-325.

15. Dimcevski G, Sami SA, Funch-Jensen P, et al. Pain in chronic pancreatitis: the role of reorganization in the central nervous system. Gastroenterology. 2007;132(4):1546-1556.

16. Sandkühler J, Benrath J, Brechtel C, Ruscheweyh R, Heinke B. Synaptic mechanisms of hyperalgesia. Prog Brain Res. 2000;129:81-100.

17. Buscher HC, Wilder-Smith $\mathrm{OH}$, van Goor H. Chronic pancreatitis patients show hyperalgesia of central origin: a pilot study. Eur J Pain. 2006;10(4):363-370.

18. Etemad B, Whitcomb DC. Chronic pancreatitis: diagnosis, classification, and new genetic developments. Gastroenterology. 2001;120(3): 682-707.

19. Ploner M, Gross J, Timmermann L, Pollok B, Schnitzler A. Pain suppresses spontaneous brain rhythms. Cereb Cortex. 2006;16(4):537-540.

20. Jongsma ML, Postma SA, Souren P, et al. Neurodegenerative properties of chronic pain: cognitive decline in patients with chronic pancreatitis. PloS ONE. 2011;6(8):e23363.

21. Jasper HH. The ten-twenty electrode system of the International Federation. Electroencephalography and Clinical Neurophysiology. 1958; 10:371-375. 
22. Gratton G, Coles MG, Donchin E. A new method for off-line removal of ocular artifact. Electroencephalogr Clin Neurophysiol. 1983;55(4): $468-484$.

23. Klimesch W, Russegger H, Doppelmayr M, Pachinger T. A method for the calculation of induced band power: implications for the significance of brain oscillations. Electroencephalogr Clin Neurophysiol. 1998;108(2):123-130.

24. Neuper C, Grabner RH, Fink A, Neubauer AC. Long-term stability and consistency of EEG event-related (de-)synchronization across different cognitive tasks. Clin Neurophysiol. 2005;116(7):1681-1694.

25. Klimesch W. EEG alpha and theta oscillations reflect cognitive and memory performance: a review and analysis. Brain Res Brain Res Rev. 1999;29(2-3):169-195.

26. Köpruner V, Pfurtscheller G, Auer LM. Quantitative EEG in normals and in patients with cerebral ischemia. Prog Brain Res. 1984;62:29-50.

27. Klimesch W, Schimke H, Pfurtscheller G. Alpha frequency, cognitive load and memory performance. Brain topogr. 1993;5(3):241-251.

28. Maltez J, Hyllienmark L, Nikulin VV, Brismar T. Time course and variability of power in different frequency bands of EEG during resting conditions. Neurophysiol Clin. 2004;34(5):195-202.
29. Posthuma D, Neale MC, Boomsma DI, de Geus EJ. Are smarter brains running faster? Heritability of alpha peak frequency, IQ, and their interrelation. Behav Genet. 2001;31(6):567-579.

30. Nir RR, Sinai A, Raz E, Sprecher E, Yarnitsky D. Pain assessment by continuous EEG: association between subjective perception of tonic pain and peak frequency of alpha oscillations during stimulation and at rest. Brain Res. 2010;1344:77-86.

31. Jeanmonod D, Magnin M, Morel A. Low-threshold calcium spike bursts in the human thalamus. Common physiopathology for sensory, motor and limbic positive symptoms. Brain. 1996;119 (Pt 2):363-375.

32. Schmidt S, Naranjo JR, Brenneisen C, et al. Pain ratings, psychological functioning and quantitative EEG in a controlled study of chronic back pain patients. PloS ONE. 2012;7(3):e31138.

33. Drewes AM, Krarup AL, Detlefsen S, Malmstrom ML, Dimcevski G, Funch-Jensen P. Pain in chronic pancreatitis: the role of neuropathic pain mechanisms. Gut. 2008;57(11):1616-1627.

34. Graversen C, Olesen SS, Olesen AE, et al. The analgesic effect of pregabalin in patients with chronic pain is reflected by changes in pharmaco-EEG spectral indices. Br J Clin Pharmacol. 2011;73(3): 363-372.
Journal of Pain Research

\section{Publish your work in this journal}

The Journal of Pain Research is an international, peer-reviewed, open access, online journal that welcomes laboratory and clinical findings in the fields of pain research and the prevention and management of pain. Original research, reviews, symposium reports, hypothesis formation and commentaries are all considered for publication.

\section{Dovepress}

The manuscript management system is completely online and includes a very quick and fair peer-review system, which is all easy to use. Visit http://www.dovepress.com/testimonials.php to read real quotes from published authors. 